\title{
Clinical Value of Hemoglobin and Albumin Levels and Lymphocyte And Platelet Count (HALP) Combination in Predicting Postoperative Complications, Lymph Node Positivity and Prognosis in Gastric Cancer Patients Who Underwent Curative Surgical Resection
}

\author{
Orçun Yalav (D, Uğur Topal (1), Ayșe Gizem Ünal (1), Ahmet Gökhan Sarıtaș (1) \\ Department of General Surgery, Çukurova University School of Medicine, Adana, Turkey
}

ORCID iDs of the authors: O.Y. 0000-000I-9239-4I63; U. T. 0000-0003-I305-2056; A.G.Ü. 0000-000I-5775-8953; A.G.S. 0000-00022039-3994.

Cite this article as: Yalav O, Topal U, Ünal AG, Sarıtaș AG. Clinical Value of Hemoglobin and Albumin Levels and Lymphocyte And Platelet Count (HALP) Combination in Predicting Postoperative Complications, Lymph Node Positivity and Prognosis in Gastric Cancer Patients Who Underwent Curative Surgical Resection. Cyprus J Med Sci 2020; 5(2): 145-52.

\section{BACKGROUND/AIMS}

In this study, we aimed to determine the clinical value of the Hemoglobin and Albumin Levels and Lymphocyte and Platelet (HALP) score in predicting postoperative complications, lymph node positivity and prognosis in patients with gastric cancer undergoing curative surgical resection.

\section{MATERIAL and METHODS}

Patients who underwent total gastrectomy for gastric adenocarcinoma between 2015-2018 were included in the study. Two groups, Groupl (lowHALP) and Group2 (highHALP), were formed. Demographic and clinical characteristics and mean survival were compared. The value of HALP score in predicting lymph node positivity and postoperative complications was evaluated at the determined cut-off value.

\section{RESULTS}

Patients were divided into two groups according to the cut-off value of 14.98. Group I consisted of 20 patients and Group 2 consisted of 62 patients. The average age in Group I was higher than Group 2 (65vs57) ( $p=0.046$ ). Female sex was higher in Group 2 than Group I $(38.7 \%$ vs $15 \%)(p=0.042)$. Total survival time was higher in Group I (4lvs28) ( $p=0.02)$. We did not find HALP score as a risk factor for survival in multivariate analysis ( $\mathrm{HR}=0.247,95 \% \mathrm{Cl}=0.113-0.485, \mathrm{p}=0.061$ ). According to the cut-off value, if the HALP value was above 14.98 , it was seen that ClavienDindo 2 and more complications developed with $84.09 \%$ sensitivity and $33.33 \%$ specificity. It is assumed that the person's lymph node is positive with a HALP value 9.14 and below, with sensitivity of $20.00 \%$ and specificity of $96.97 \%$.

\section{CONCLUSION}

Our findings showed that HALP is closely related to clinicopathological features but it is not an independent prognostic factor for survival. Its value in predicting the risk of complication development and lymph node positivity is limited.

Keywords: Gastric cancer, HALP score postoperative complications, prognosis

\section{INTRODUCTION}

Gastric cancer is the fourth most common cancer and the second most common cause of cancer-related deaths (I). The incidence in our country is approximately 6.3-14.2 per hundred thousand. It ranks $2^{\text {nd }}$ in cancer related deaths in men and 4th in women in the world (2). Gastric cancer is still an important health problem in terms of incidence and prognosis. Knowledge of prognostic factors in gastric cancer is important for determining survival and optimal treatment strategies.

Tumor, nodes, and metastases (TNM) stage and distant metastasis of tumor have been accepted as the primary factor in determining prognosis (3). The disadvantage of TNM staging in determining prognosis is that it only reflects the characteristics of cancer. In particular, the outcomes of some patients at the same stage may be completely different. Therefore, other factors should be considered in predicting prognosis in patients with gastric cancer. Recently, various 
predictions have been defined and applied to predict the shortand long-term outcomes of gastric cancer patients. These predictions include cancer-related factors, host-related factors, surgical-related factors, and systemic inflammatory response markers (4-9).

Systemic inflammation and nutritional status play an important role in the prognosis of cancer patients (I0). Hemoglobin and albumin levels are commonly used markers to assess nutritional status and performance of the patient. Anemia and low albumin were associated with poor prognosis in cancer (II, I2). In previous studies, increased preoperative platelet count was associated with increased recurrence, serosal invasion and advanced stage of gastric cancer (I3). Lymphocytes play an important role in defense against cancer by inducing cytotoxic cell death, inhibiting cancer cell proliferation and migration (14). Based on this evidence, several inflammatory index combinations such as neutrophil-lymphocyte ratio (NLR), platelet-lymphocyte ratio (PLR) HALP combination and systemic immune-inflammation index have been used to predict prognosis (15-19).

In recent studies, a new composite index named HALP, calculated as Hemoglobin $(\mathrm{g} / \mathrm{L}) \times$ Albumin $(\mathrm{g} / \mathrm{L}) \times$ Lymphocyte $(/ \mathrm{L})$ / Platelet (/L) was reported to be related to survival in gastric cancer (19), colorectal cancer (20), bladder cancer (21), and renal cancer (22) patients. Currently, there is no study on the relationship between HALP and postoperative complications in gastric cancer and its ability to predict lymph node positivity.

In this study, we aimed to determine the relationship between preoperative hemoglobin, albumin level and lymphocyte and platelet count (HALP) combination with prognosis, postoperative complications and lymph node positivity in patients with gastric cancer undergoing curative resection.

\section{MATERIAL AND METHODS}

\section{Study Population and Data Collection}

120 patients who underwent curative surgery for gastric cancer in Cukurova Medical Faculty General Surgery Clinic, between January 2015 and December 2018, were included in the study. Permission was obtained from Cukurova University Faculty of Medicine Ethics Committee dated 04.09.2019 and numbered 91/26. Patient files and hospital information system records were examined prospectively, and a database was created. Patients were analyzed retrospectively using this database. Patients with stage IV gastric cancer, patients under eighteen years of age, pregnant patients, patients with chronic inflam-

\section{Main Points:}

- Nutritional and immune status is important to the prognosis of patients with gastric carcinoma.

- HALP score is closely related to clinicopathological features but it is not an independent prognostic factor for survival.

- We did not find any correlation between HALP score and risk of postoperative complications.

- HALP score is closely related to clinicopathological features. matory disease (Tuberculosis, Sarcoidosis, etc.), patients who underwent palliative surgery, patients with autoimmune disease, patients with hematologic disease, who were using steroids for any reason and whose records could not be accessed were excluded from the study. The remaining 82 patients were included in the study.

After the cut-off value was determined by ROC curves, the patients were divided into two groups according to the cut-off value as Group I (low HALP) and Group 2 (high HALP). Demographic characteristics, Body Mass Index (BMI), comorbidities, American Society of Anesthesiologists (ASA) score, neoadjuvant treatment status, type and nature of the operation, tumor localization and tumor pathologic stage were recorded. Pathological stage of the tumor, total and metastatic lymph node number, operation duration, mean blood loss, conversion rate in laparoscopic cases, mean time to start oral intake, postoperative complication status according to Clavien Dindo classification (23), rate of anastomosis leakage, postoperative hospital stay, 30-day mortality, unplanned re-admission rate and total survival rate in the postoperative 30-day period were recorded and this information was compared between the two groups. The clinical value of the HALP score in predicting postoperative complications and lymph node positivity was calculated.

Tumor-node-metastasis (TNM) 2016 system was used for tumor staging.

Anastomosis leakage was defined as a deterioration of anastomosis integrity determined by combination of clinical, radiological and operative tools.

Wound infection was defined as superficial or deep incisional surgical site infection in the surgical wound according to the definition of the Centers for Disease Control (CDC) (24).

Tumor invasion depth was evaluated preoperatively by endoscopic ultrasound in suspected cases. Contrast-enhanced thorax, upper and lower abdominal computed tomography were performed for staging and Positron Emition Tomography (PETCT) was performed for metastasis screening

The total blood count was measured by an automated hematology analyzer (Roche Hitachi Cobas ${ }^{\circledR} 8000$ Roche Diagnostics, Indianapolis, IN, USA). While calculating the HALP index, hemoglobin $(\mathrm{g} / \mathrm{L})$, albumin $(\mathrm{g} / \mathrm{L})$, lymphocytes $(/ \mathrm{L})$, platelets $(/ \mathrm{L})$ units conversion was performed in normal value units.

\section{Statistical Analyses}

Data were analyzed using IBM Statistical Package for the Social Sciences for Windows, version 24 (IBM Corp.; Armonk, NY, USA). When evaluating the data of the study, in addition to descriptive statistical methods (mean, standard deviation, median, frequency, ratio, minimum, maximum), Student's t test was used for the comparison of quantitative data and Mann Whitney $U$ test was used for the evaluation of neutrophil/lymphocyte ratio which did not show normal distribution. Pearson's Chi-squared test and Fisher's Exact test were used to compare qualitative data, and logistic regression was used for multivariate evaluations. The patients were divided into two groups according to survival and cut off value was found by ROC analysis. The cut- 
off value for lymph node positivity was calculated by dividing the cases into lymph node positive and negative groups. Diagnostic accuracy was evaluated using receiver operating characteristic (ROC) curve analysis Appropriate cut-off values were identified, and sensitivity, specificity, positive predictive value, negative predictive value, positive likelihood ratio, and negative likelihood ratio were calculated for parameters with an area under the curve (AUC) of above 0.600. To assess the association of HALP with gastric cancer overall survival, multivariate Cox's proportional hazard model was conducted to estimate Hazard ratios (HRs) and their 95\% confidence intervals ( $\mathrm{Cls}$ ). Kaplan-Meier and Log Rank tests were used for survival analysis. A p value of $<0.05$ was considered statistically significant.

\section{RESULTS}

In order to create a cut-off value for HALP, ROC analysis and $\mathrm{ROC}$ curve were created. As a result of ROC analysis, the area under the ROC curve was calculated as $50.3 \%$. The obtained

TABLE I. Proposed cut-off values for significant parameters in postoperative complications, Clavien Dindo 2 and above

\begin{tabular}{lc|} 
& HALP \\
\hline AUC & 0.503 \\
Cut-off & $>14.98$ \\
Specifity & 33.33 \\
$95 \%-C l(\%)$ & $19.1-50.2$ \\
Sensitive (\%) & 84.09 \\
$95 \%-C l(\%)$ & $69.9-93.4$ \\
PPV & 58.7 \\
NPV & 65.0 \\
+LR & 1.26 \\
-LR & 0.48 \\
P & 0.958 \\
\hline AUC: Area under the curve; PPV: Positive predictive value; NPV: Neg- \\
ative predictive value; OR: Odds ratio; +LR: Positive likelihood ratio; -LR: \\
Negative likelihood ratio; HALP: Hemoglobin and Albumin Levels and \\
Lymphocyte and Platelet
\end{tabular}

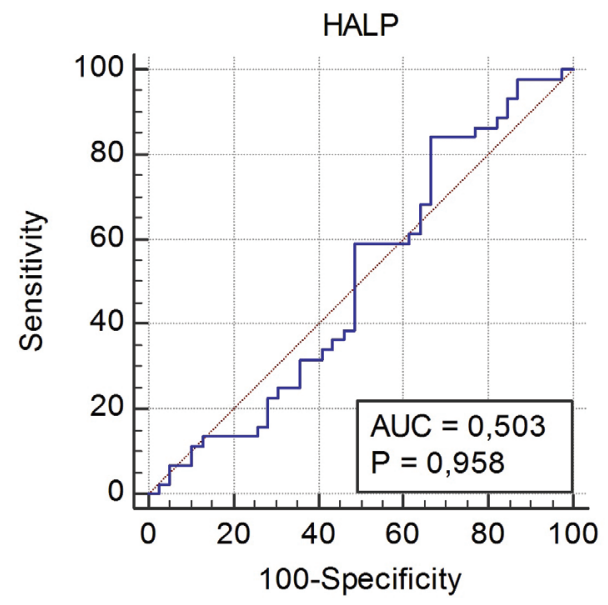

FIGURE I. Receiver operating characteristic (ROC) curve analyses for postoperatif complications cut-off value gives a correct answer at a rate of 50.3\%. According to the cut-off value, if the HALP value was above 14.98, it was seen that Clavien Dindo 2 and more complications developed with $84.09 \%$ sensitivity and $33.33 \%$ specificity. The findings of this evaluation are shown in Table I and Figure I.

In order to create a cut-off value for HALP, ROC analysis and $\mathrm{ROC}$ curve were created. As a result of ROC analysis, the area under the ROC curve was calculated as $50.3 \%$. The obtained cut-off value gives a correct answer at a rate of 50.3\%. According to the cut off value obtained, the lymph node positivity is assumed to be positive for a HALP value of 9.14 and below, with a sensitivity of $20.00 \%$ and specificity of $96.97 \%$. It is shown in Table 2 and Figure 2.

Patients were divided into two groups according to the cut-off value of 14.98. Group I consisted of 20 patients and Group 2 consisted of 62 patients. The average age in Group I was higher than

\begin{tabular}{lc} 
TABLE 2. Proposed cut-off values for significant parameters in lymph \\
node positivity & HALP \\
\hline AUC & 0.503 \\
Cut-off & $<9.14$ \\
Specificity & 96.97 \\
95\%-Cl (\%) & $84.2-99.9$ \\
Sensitive (\%) & 20.0 \\
95\%-Cl (\%) & $10.0-33.7$ \\
PPV & 90.9 \\
NPV & 44.4 \\
+LR & 6.60 \\
-LR & 0.83 \\
P & 0.962 \\
AUC: Area under the curve; PPV: Positive predictive value; NPV: Neg- \\
ative predictive value; OR: Odds ratio; +LR: Positive likelihood ratio; - LR: \\
Negative likelihood ratio; HALP: Hemoglobin and Albumin Levels and \\
Lymphocyte and Platelet
\end{tabular}

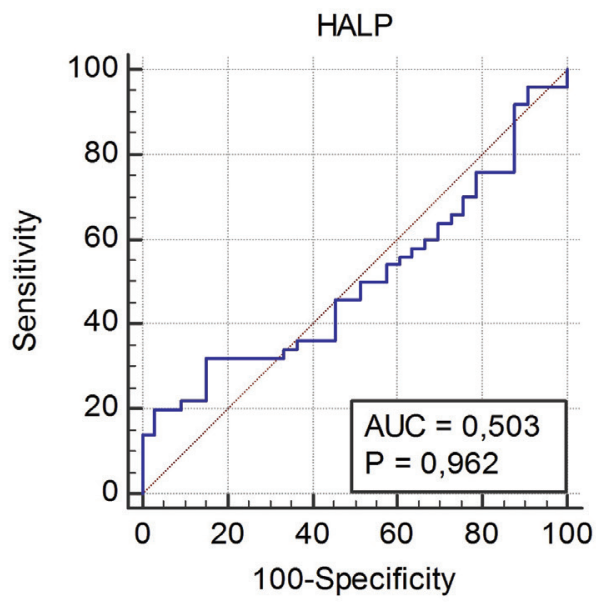

FIGURE 2. Receiver operating characteristic (ROC) curve analyses for lymph node positivity 
Group 2 (65 vs 57) ( $p=0.046)$. Female sex was higher in Group 2 than Group I (38.7\% vs 15\%) ( $p=0.042)$. Body mass index was higher in Group 2 than Group I (25.4 vs 22.9l) ( $p=0.020$ ). No statistically significant difference was found between the groups in terms of ASA scores and neoadjuvant treatment ( $p>0.05)$. Demographic characteristics and preoperative findings of the patients are shown in Table 3.

In both groups, conventional surgical operations were performed more frequently ( $95 \%$ vs $82.3 \%$ ) than other surgical techniques $(p=0.149)$. Duration of operation was similar in groups (2188 vs $232 \mathrm{~min}, \mathrm{p}=0.385$ ). Postoperative complication rates were similar among the groups according to the Clavien Dindo classification $(p=0.298)$. Anastomotic leakage rates were similar in both groups ( $p=0.692$ ). Postoperative mortality rates were similar in both groups (20\% vs 8.1\%; $p=0.142$ ). Postoperative hospital stay was similar among the groups $(p=0.157)$. The most common reason for admission to the hospital within 30 days after discharge was wound infection ( $10 \%$ vs $9.7 \%, p=0.134$ ). Intraoperative and postoperative outcomes are shown in Table 4.

Tumor localization was most commonly in the antrum in both groups ( $p=0.646)$. The total number of dissected lymph nodes was similar in the groups (30 vs 29) $(p=0.876)$. The number of positive lymph nodes was significantly higher in Group I than Group 2 ( 14 vs 5; $p=0.002$ ). The rate of lymph node positivity was similar in the groups $(p=0.089)$. The pathological stage was similar in the groups $(p=0.110)$. The rate of well-differentiated tumors was lower in Group I ( $5 \%$ vs 29\%; $p=0.047$ ). The pathological features of the tumors are shown in Table 5.

\begin{tabular}{|c|c|c|c|c|}
\hline & & Group I Low HALP & Group2 High HALP & $p^{*}$ \\
\hline Age (min-max) & & $65,30+17,82(14-89)$ & $57.53+|3.9|(28-85)$ & 0.046 \\
\hline Sex & Male & $17(85.0)$ & $38(61.3)$ & \\
\hline \multirow[t]{2}{*}{ ASA score } & । & $12(60.0)$ & $33(53.2)$ & 0.449 \\
\hline & 3 & $4(20.0)$ & $8(12.9)$ & \\
\hline BMI (min-max) & & $22,91+2,41(19-28.6)$ & $25.4 \pm 4.47(16-40.3)$ & 0.020 \\
\hline \multirow[t]{2}{*}{ Neoadjuvant Chemotherapy } & No & $16(80.0)$ & $43(69.4)$ & 0.268 \\
\hline & Yes & $4(20.0)$ & $19(30.6)$ & \\
\hline
\end{tabular}

\begin{tabular}{|c|c|c|c|c|}
\hline & & Group I Low HALP & Group2 High HALP & $\mathrm{p}^{*}$ \\
\hline Operation type & Open & $19(95.0)$ & $51(82.3)$ & 0.149 \\
\hline Operation duration (min) & & $218.25 \pm 29.03(180-310)$ & $232.50 \pm 70.76(170-500)$ & 0.385 \\
\hline \multirow[t]{4}{*}{ Complication (Clavien Dindo) } & 1 & I (5.0) & $14(22.6)$ & \multirow[t]{4}{*}{0.298} \\
\hline & $3 \mathrm{~A}$ & $3(15.0)$ & $9(14.5)$ & \\
\hline & 3B & I (5.0) & $2(3.2)$ & \\
\hline & 5 & $3(15.0)$ & $3(4.8)$ & \\
\hline Anastomosis leakage & None & $17(85.0)$ & $55(88.7)$ & 0.692 \\
\hline Postoperative mortality & No & $16(80.0)$ & $57(91.9)$ & 0.142 \\
\hline Postoperative duration of hospitalization (day) & $|4.0+1| .88(5-45)$ & $10.79+7.48(2-46.0)$ & 0.157 & \\
\hline \multirow[t]{5}{*}{ 30-day readmission to the hospital } & None & $15(75.0)$ & $55(88.7)$ & \multirow[t]{5}{*}{0.123} \\
\hline & Ileus & I (5.0) & I (I.6) & \\
\hline & Oral intake disorder & I (5.0) & $0(0.0)$ & \\
\hline & Pneumonia & I (5.0) & $0(0.0)$ & \\
\hline & Wound site infection & $2(10.0)$ & $6(9.7)$ & \\
\hline
\end{tabular}




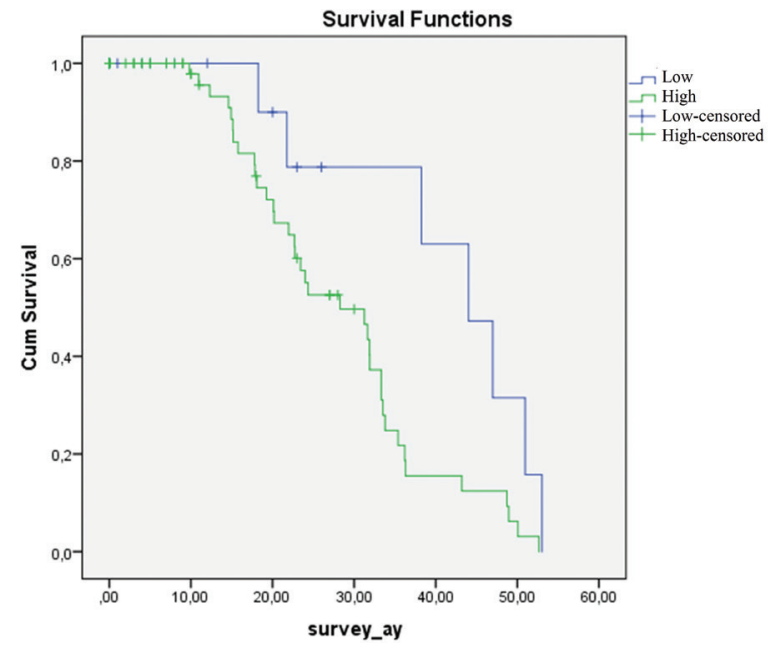

FIGURE 3. Total survival time according to HALP groups
Total survival time was higher in Group I (4I vs 28; $p=0.02$ ). It is shown in Table 6 and Figure 3.

There were statistically significant differences in univariate and multivariate analyzes in terms of age and pathological grade groups $(p<0.01)$. There was no statistically significant difference between the patients in terms of sex, pathological stage, HALP group, lymph node positivity and tumor localization ( $p>0.05)$. Univariate and multivariate analyzes of the variables of age, sex, pathological grade, pathological stage, HALP score level, lymph node positivity and tumor localization are shown in Table 7.

\section{DISCUSSION}

Proper prognostic evaluation is necessary for the optimal treatment of gastric cancer. TNM staging system plays an important role in the prognostic evaluation of gastric cancer in routine clinical practice. However, clinical outcomes may vary between pa-

\begin{tabular}{|c|c|c|c|c|}
\hline & & Group I Low HALP & Group2 High HALP & $\mathrm{p}^{*}$ \\
\hline \multirow[t]{4}{*}{ Tumor localization } & Antrum & $6(30.0)$ & $25(40.3)$ & \multirow[t]{4}{*}{0.646} \\
\hline & Corpus & $7(35.0)$ & $19(30.6)$ & \\
\hline & Small curvature & $3(15.0)$ & $9(14.5)$ & \\
\hline & EGJ & $0(0.0)$ & $2(3.2)$ & \\
\hline Total dissected lymph node number (mean) (min-max) & & $30.35+15.85(3-62)$ & $29.79+13.16(7-63)$ & 0.876 \\
\hline Positive lymph node number (mean) (min-max) & & $14.05+15.94(0-47)$ & $5.54+7.77(0-38)$ & 0.002 \\
\hline Lymph node positivity & Negative & $5(25.0)$ & $28(45.2)$ & 0.089 \\
\hline \multirow{5}{*}{ pSTAGE } & $2 \mathrm{~A}$ & I (5.0) & $4(6.5)$ & \multirow{5}{*}{0.110} \\
\hline & $2 \mathrm{~B}$ & $4(20.0)$ & $16(25.8)$ & \\
\hline & $3 \mathrm{~A}$ & I (5.0) & $7(11.3)$ & \\
\hline & $3 B$ & $3(15.0)$ & $3(4.8)$ & \\
\hline & $3 C$ & $10(50.0)$ & $15(24.2)$ & \\
\hline \multirow[t]{3}{*}{ Pathological grade } & Non-differentiated & $3(15.0)$ & II (17.7) & \multirow[t]{3}{*}{0.047} \\
\hline & Poorly differentiated & $9(45.0)$ & $25(40.3)$ & \\
\hline & Mildly differentiated & $7(35.0)$ & $8(12.9)$ & \\
\hline
\end{tabular}

TABLE 6. Total survival time according to HALP groups

\begin{tabular}{|c|c|c|c|c|}
\hline \multirow{3}{*}{ HALP Group } & & $32.54-49.47$ & $33.68-54.37$ & 0.020 \\
\hline & High HALP & $28.44+1.86$ & $28.26+5.15$ & \\
\hline & & $24.8-32.09$ & $18.16-38.37$ & \\
\hline
\end{tabular}


tients with the same staging process, and this is evidence that the TNM staging system is not sufficient to predict prognosis (25-27). Due to the insufficiency of the TNM staging system in determining the prognosis, researchers have begun to work on new scoring systems.

It is widely accepted that inflammatory response and nutritional status are associated with the prognosis of cancer patients (4). Serum albumin is one of the most commonly used indicators to predict the nutritional status of the patient. Serum albumin level is one of the parameters used to evaluate cancer progression and prognosis. Low albumin levels in cancer patients are correlated with low survival rates (28). Anemia is a common finding in cancer patients and is considered a negative prognostic factor (II). Lymphopenia is also common in advanced cancer patients and is a warning for cancer progression. Metastasis formation is associated with platelet stimulation. Platelets are thought to protect cancer cells from immunological attacks (2931). HALP is an integration of these four hematological and biochemical parameters and has been shown to have a prognostic value in patients with gastric cancer (19).
In this study, the HALP score was calculated based on preoperative hemoglobin, albumin, lymphocyte and platelet values and its importance in the prognostic evaluation of gastric cancer was evaluated.

In this study, the clinical significance of the HALP score in predicting cases with a score of 2 or more according to the Clavien Dindo scoring system evaluating postoperative complications was investigated. At a 14.98 cut off value, the sensitivity in detecting complications was as high as $84 \%$, while the specificity was very low at $33 \%$. When used to estimate lymph node positivity, the specificity of detecting lymph node positivity at a cutoff value of 9.14 was very high $(96.7 \%)$, whereas its sensitivity was low (20\%).

Chen XL et al. (19) found the HALP score to be closely related to many clinicopathological features such as tumor diameter, $T$ and $\mathrm{N}$ stage, and vascular invasion. They found the HALP score as a risk factor for survival in multivariate analyzes, and the median survival time and overall survival rates for l, 2, 3 years were higher in the high HALP group. In our study, it was associated with age, sex, and body mass index. We did not find any relationship

\begin{tabular}{|c|c|c|c|c|}
\hline \multirow[b]{2}{*}{ Measurements } & & \multirow{2}{*}{$\frac{\text { Univariate }}{p}$} & \multicolumn{2}{|c|}{ Multivariate } \\
\hline & & & $\mathrm{HR}(95 \%-\mathrm{Cl})$ & $p$ \\
\hline Age group & $>59$ & & $0.258(0.076-0.441)$ & \\
\hline Sex & Male & 0.051 & 1.00 & 0.058 \\
\hline \multirow[t]{4}{*}{ Pathological grade } & Poorly differentiated & 0.046 & 1.00 & 0.039 \\
\hline & Non-differentiated & & & \\
\hline & Well differentiated & & & \\
\hline & Mildly differentiated & & $0.414(0.126-0.702)$ & \\
\hline \multirow{4}{*}{ Pathological stage } & $2 \mathrm{~B}$ & & & \\
\hline & $3 A$ & & $0.233(-0.147-0.613)$ & \\
\hline & $3 B$ & & & \\
\hline & $3 C$ & & & \\
\hline \multirow[t]{2}{*}{ HALP } & $<14.98$ & 0.055 & 1.00 & 0.061 \\
\hline & $>14.98$ & & $0.247(0.113-0.485)$ & \\
\hline \multirow[t]{2}{*}{ Lymph node positivity } & Negative & 0.113 & 1.00 & 0.148 \\
\hline & Positive & & $0.155(-0.037-0.346)$ & \\
\hline Tumor localization & Antrum & 0.664 & 1.00 & 0.545 \\
\hline
\end{tabular}


between intraoperative and postoperative outcomes. Contrary to the findings of Chen XL et al. (19), it was not associated with pathological tumor stage. However, the rate of positive lymph nodes was high, especially in the group with low HALP scores (14 vs $5 ; p=0.002$ ). The pathological grade had a higher proportion of well differentiated tumors in the group with high HALP (29\% vs $5 \% ; p=0.047$ ).

We did not find the HALP score as a risk factor for survival in the multivariate analysis. The mean total survival time was higher in the low HALP group, contrary to expectations ( 4 I months vs 28 months; $\mathrm{p}=0.020$ ).

The most important limitation of our study was its retrospective evaluation and single-centeredness. However, we believe that it provides comprehensive data and contributes to valuable reference data in terms of HALP score's value in predicting lymph node positivity in gastric cancer. Multicenter prospective studies are needed to confirm our findings.

In our study contrary to what I expected to have a low Halp score, we did not find it related to poor prognosis. Preoperative high HALP score was found to be associated with poor prognosis. We did not find any correlation between HALP score and risk of postoperative complications. The HALP score is an easy-toaccess and inexpensive biomarker. However, it cannot be used as a prognostic factor alone in gastric cancer. The. Prognostic tools are needed to create personalized cancer treatment programs.

Ethics Committee Approval: Ethics committee approval was received for this study from the ethics committee of Cukurova University Faculty of Medicine (04.09.2019/91/26).

Informed Consent: Due to the retrospective design of the study, informed consent was not taken.

Peer-review: Externally peer-reviewed.

Author Contributions: Concept - O.Y., U.T., A.G.Ü., A.G.S.; Design - O.Y., U.T., A.G.Ü.; Supervision - O.Y.; Resources - O.Y., U.T., A.G.Ü., A.G.S.; Materials - O.Y., U.T., A.G.Ü., A.G.S.; Data Collection and/or Processing U.T., A.G.Ü.; Analysis and/or Interpretation - O.Y., U.T.; Literature Search - O.Y., U.T., A.G.Ü., A.G.S.; Writing Manuscript - O.Y., U.T.; Critical Review - O.Y., U.T.

Conflict of Interest: Authors have no conflicts of interest to declare.

Financial Disclosure: The authors declared that this study has received no financial support.

\section{REFERENCES}

I. Torre LA, Bray F, Siegel RL, Ferlay J, Lortet-Tieulent J, Jemal A. Global cancer statistics, 2012. CA-Cancer J Clin 2015; 65(2): 87-I08. [Crossref]

2. Türkiye'de Kanser İstatistikleri. Available From: URL: https://hsgm. saglik.gov.tr/depo/birimler/kanser-db/istatistik/Turkiye_Kanser_Istatistikleri_2015.pdf_Accessed September ,10, 2019.

3. Ajani JA, Bentrem DJ, Besh S, D'Amico TA, Das P, Denlinger $C$, et al. Gastric cancer version 2.2013: featured updates to the NCCN Guidelines. J Natl Compr Canc Netw 20I3; II(5): 53I-46. [Crossref]

4. Hsieh MC, Wang SH, Chuah SK, Lin YH, Lan J, Rau KM. A prognostic model using inflammation-and nutrition-based scores in patients with metastatic gastric adenocarcinoma treated with chemotherapy. Medicine 2016; 95(17): I-6. [Crossref]

5. Roxburgh CS, McMillan DC. Role of systemic inflammatory response in predicting survival in patients with primary operable cancer. Future Oncol 20I0; 6(I): 149-63. [Crossref]

6. Mohri Y, Tanaka K, Ohi M, Yokoe T, Miki C, Kusunoki M. Prognostic significance of ost- and tumor-related factors in patients with gastric cancer. World J Surg 20I0; 34(2): 285-90. [Crossref]

7. Xin-Ji Z, Yong-Gang L, Xiao-Jun S, Xiao-Wu C, Dong Z, Da-Jian Z The prognostic role of neutrophils to lymphocytes ratio and platelet count in gastric cancer: A meta-analysis. Int J Surg 2015; 21: 84-91. [Crossref]

8. D'Amata G, Izzo L, Pugliese F, Izzo S, Izzo P, Costi U, et al. New prognostic factors in gastric cancer: the role of lympho-plasmacytic infiltrate. Annali italiani di chirurgia 2018; 89(5): 398-405.

9. Del Rio P, Viani L, Bertocchi E, lapichino G, Luzietti E, Dell'Abate P, et al. The prognostic role of tumor size in patients with gastric cancer. Annali italiani di chirurgia 2017; 88(6): 478-84.

10. Pastore CA, Orlandi SP, González M. CAssociation between an inflammatory-nutritional index and nutritional status in cancer patients. Nutricion Hospitalaria 20132; 28(I): 188-93.

II. Huang XZ, Yang YC, Chen Y, Wu CC, Lin RF, Wang ZN, et al. Preoperative anemia or low hemoglobin predicts poor prognosis in gastric cancer patients: a meta-analysis, Dis Markers 2019; 7606128: I-9. [Crossref]

12. Ouyang $X$, Dang $Y$, Zhang F, Huang Q. Low Serum Albumin Correlates with Poor Survival in Gastric Cancer Patients. Clin Lab 2018; 64(3): 239-45. [Crossref]

13. Yang $C$, Jiang $H$, Huang $S$, Hong $H$, Huang $X$, Wang $X$, et al The prognostic role of pretreatment thrombocytosis in gastric cancer: A systematic review and meta-analysis. Medicine (Baltimore) 2018; 97(31): 1-77. [Crossref]

14. Mantovani A, Allavena P, Sica A, Balkwill F. Cancer-related inflammation. Nature 2008; 454: 436-44. [Crossref]

15. Szor DJ, Dias AR, Pereira MA, Ramos MFKP, Zilberstein B, Cecconello, et al. Prognostic role of neutrophil/lymphocyte ratio in resected gastric Cancer: a systematic review and meta-analysis Clinics 2018; 73: e360. [Crossref]

16. Yalav O, Topal U, Ünal AG. Clinical value of neutrophil/lymphocyte ratio in predicting postoperative complications, lymph node positivity and prognosis in gastric cancer patients who underwent curative surgical resection Ann Med Res 2019; 26(II): 2513-9. [Crossref]

17. Miyamoto R, Inagawa S, Sano N, Tadano S, Adachi S, Yamamoto M. The neutrophil-to-lymphocyte ratio (NLR) predicts short-term and long-term outcomes in gastric cancer patients. Eur J Surg Oncol 2018; 44(5): 607-I2. [Crossref]

18. Dolan RD, Lim J, McSorley ST, Horgan PG, McMillan DC. The role of the systemic inflammatory response in predicting outcomes in patients with operable cancer: systematic review and meta-analysis. Sci Rep 2017; 7(I): I-31. [Crossref]

19. Chen XL, Xue L, Wang W, Chen HN, Zhang WH, Liu K, et al. Prognostic significance of the combination of preoperative hemoglobin, albumin, lymphocyte and platelet in patients with gastric carcinoma: a retrospective cohort study. Oncotarget 2015; 6(38): 41370-82. [Crossref]

20. Jiang H, Li H, Li A, Tang E, Xu D, Chen Y, et al. Preoperative combined hemoglobin, albumin, lymphocyte and platelet levels predict survival in patients with locally advanced colorectal cancer. Oncotarget 2016; 7(4): 72076-83. [Crossref]

21. Peng D, Zhang CJ, Gong YQ, Hao H, Guan B, Li XS, et al. Prognostic significance of HALP (hemoglobin, albumin, lymphocyte and platelet) in patients with bladder cancer after radical cystectomy. Sci Rep 2018; 8(I): I-9. [Crossref]

22. Peng $D$, Zhang $C J$, Tang $Q$, Zhang $L$, Yang KW, Yu XT, et al. Prognostic significance of the combination of preoperative hemoglobin and albumin levels and lymphocyte and platelet counts (HALP) in pa- 
tients with renal cell carcinoma after nephrectomy. BMC Urol 2018; I8(I): 20. [Crossref]

23. Lee KG, Lee HJ, Yang JY, Oh SY, Bard S, Suh YS, et al. Risk factors associated with complication following gastrectomy for gastric cancer: retrospective analysis of prospectively collected data based on the Clavien-Dindo system. J Gastrointest Surg 2014; 18: 1269-77. [Crossref]

24. Horan TC, Gaynes RP, Martone WJ, Jarvis WR, Emori TG. CDC definitions of nosocomial surgical site infections, 1992: a modification of CDC definitions of surgical wound infections. Am J Infect Control 1992; 13(10): 606-8. [Crossref]

25. McLean MH, El-Omar EM. Genetics of gastric cancer. Nat Rev Gastroenterol Hepatol 2014; II: 664-74. [Crossref]

26. Galon J, Pagès F, Marincola FM, Thurin $M$, Trinchieri G, Fox BA, et al. The Immune Score as a new possible approach for the classification of cancer. J Transl Med 20I2; IO(I): I-4. [Crossref]
27. Jiang Y, Zhang $Q, H u$ Y, Li T, Yu J, Zhao L, et al. ImmunoScore signature: a prognostic and predictive tool in gastric cancer. Ann Surg 2018; 267(3): 504-13. [Crossref]

28. Yao ZH, Tian GY, Yang SX, Wan YY, Kang YM, Liu Q. H et al Serum albumin as a significant prognostic factor in patients with malignant pleural mesothelioma. Tumor Biol 20l4; 35(7): 6839-45. [Crossref]

29. Schlesinger $M$. Role of platelets and platelet receptors in cancer metastasis. J Hematol Oncol 20I8; II(I): I-I5. [Crossref]

30. Haemmerle M, Stone RL, Menter DG, Afshar-Kharghan $\bigvee$, Sood AK. The platelet lifeline to cancer: challenges and opportunities. Cancer Cell 2018; 33(6): 965-83. [Crossref]

31. Stegner D, Dutting S, Nieswandt B. Mechanistic explanation for platelet contribution to cancer metastasis. Thromb Res 20l4; 133(2): 149-57. [Crossref] 